G. H. Miley, W. R. Sutton III, and D. J. Sigmar, submitted to 12 th European conference on Controlled-Fusion Research and Plasma Physics, Budapest, Hungary, Sept. 2-6, 198.

\title{
AL.PHA-DRIVEN FAST MAGNETOSONIC WAVE HEATING IN TOKAMAK PLASMAS
}

George H. Miley, University of I11inois Fusion Studies Laboratory, Urbana, I11ino is 61801, William R. Sutton III,* and Dieter J. Sigmar**

An alpha-driven fast magnetosonic wave instability is investigated in tokamak plasmas for propagation transverse to the external magnetic field at frequencies several times the alpha gyrorate. A two-dimensional differential quasi-linear diffusion equation is derived in cylindrical $v_{\perp}-v_{\| l}$ geometry. The quasi-1inear diffusion coefficients in the smal? parameter $k_{\|} k_{\perp}$ are expanded and the problem is reduced to one dimension by integrating out the $v_{\|}$dependence. Reactor relevant information is obtained using data from the one-dimensional formulation in a 1-1/2dimensional tokamak transport code. Contour plots of the alpha threshold fraction are used to identify the instability regions in the $n_{e}-T_{j}$ plane. Alpha/background electron fractions as $10 \mathrm{w}$ as $10^{-6}$ to $10^{-4}$ may trigger the instability. For a typical reactor-size tokamak, an enhancement of the fraction of the alpha energy transferred to ions by as much as 1.5 can occur for $T_{i}=T_{e}$ at $7 \mathrm{keV}$. Still, due to the rapid equilibration of electron and ion temperatures, a $<1$ to $2 \%$ increase in fusion power occurs overal1.

$$
\text { CONF-850927--3 }
$$

\section{DE85 014279}

\footnotetext{
* Present address: Southern Company Services, P. 0. Box 2625, Birmingham, Alabama 35202. **Present address: Oak Ridge National Laboratory, Fusion Energy Division, P. 0. Box Y, Oak Ridge, Tennessee 37831.
}

\section{DISCLAIMER}

\begin{abstract}
This report was prepared as an account of work sponsored by as agency of the L'nited States Government. Neither the United States Government nor any agency thereof, nor any of their employees, makes any warranty, express or implied, o: assumes any legal liability or responsibility for the accuracy, completeness, or usefulness of any information, afparatus, product, or process disclosed, or represents that its use would not infringe privately owned rights. Reference herein to any specific cornmercial product, process, or service by trade neme, trademark, manufacturer, or otherwise dces not necessarily constitute or imply its cildorsement, recommendation, or faworing by the Unitad States Government or any agency thereof. The views and opinions of author exprosed berein do not nesessarily state or reflect those of the United States Government or any agency thereof.
\end{abstract}


G. H. Miley, W. R. Sutton III, and D. J. Sigmar, submitted to 12th European Conference on Controlzed Fusion Research and Plasma Physics, Budapest, Hungary, Sept. 2-6; 1985

\section{ALPHA-DRIVEN FAST MAGNETOSONIC WAVE HEATING IN TOKAMAK PLASHAS}

George H. Miley, William R. Sutton, University of Illinois Fusion Studies Laboratory, Urbana, I1linois 61801, USA, and Dieter J. Sigmar, Oak Ridge Mational Laboratory, Oak Ridge, TN 37830 , USA

Abstract--An alpha-driven fast magnetosonic wave instability is investigated in tokamaks. Contour plots of the alpha threshold fraction are used to identify instability reyions in the $n_{e^{-T_{j}}}$ piane.

Introduction--We investigate the potential effect of an alpha-particle-driven fast magnetosonic (FMS) wave instability ${ }^{-4}$ in a D-T fueled tokamak. A perpendicularly propagating $\left(k_{n}<<k_{1}\right)$ FMS wave is assumed. We employ an isotropic alpha birth distribution to calculate the energy released during quasi-linear diffusion in velocity space. Results are used in a $1 \frac{1 / 2-D}{-0}$ ransport code to scope potential reactor effects.

Quasi-Linear Diffusion--We take an FMS wave propagating at an arbitrary anyle to the applied toroidal magnetic field, and employ circular cylindrical $v_{1}-v_{1}$ coordinates. We approximate a cylindrical tokamak with Cartesian geometry $(r \gg>1 / k 1)$. With $\hat{x}=\hat{r}, \hat{y}=\hat{\theta}$, and $\hat{z}=\hat{z}$, the general quasi-linear diffusion equation is: ${ }^{5}$ (nomenclature follows Ref 4 )

$$
\begin{aligned}
& \frac{\partial f_{\alpha}}{\partial \tau}=\frac{m_{\alpha}^{2}}{m_{\alpha}} \sum \delta d \vec{k} \frac{1}{v_{\perp}} \hat{R}_{j}\left\{[\ldots]\left(\hat{R}_{2} f_{\alpha}\right) \delta\left(\omega(\vec{k})-L \Omega_{\alpha}-k_{n} v_{n}\right)\right\} \text {. } \\
& \hat{\mathrm{R}}_{1}=\hat{\mathrm{R}}_{2}=\left(1-\frac{k_{11} v_{n}}{\omega}\right) \frac{\partial}{\partial v_{\perp}}+\frac{k_{11} v_{2}}{\omega} \frac{\partial}{\partial v_{n}} \\
& {[\ldots]=v_{\perp}\left|E_{r} \frac{L}{|\lambda|} J_{L}+i \eta_{c} E_{\theta} J_{L}^{\prime}+\frac{v_{u}}{v_{\perp}} E_{2} J_{L}\right|^{2}} \\
& \lambda=k_{\perp} v_{\perp} / \Omega_{\alpha} \quad \eta_{\propto}=q_{\propto} /\left|q_{\alpha}\right| \quad J_{L}=J_{L}(1 \lambda 1)
\end{aligned}
$$

where $\varepsilon_{1}, \varepsilon_{2}$, and $\varepsilon_{X}$ are elements of the dielectric tensor. (We reject the second solution to $\Delta=0$ for $\vec{k}=k_{1}$ ) Equation 2 is formally inverted to give $\omega=\omega(k)$. We approximate the spectral density of the electric field by a box function in $k$ space and substitute these forms into Eq. (1) and associate the delta function with the integral over $k_{1}$.

Diffusion Coefficients--Next, we expand the Bessel functions in the small parameter $k_{n} v_{n} / L \Omega_{\alpha}$, perform the inteyral over $k_{n}$ exactly and expand the evaluated integral again in the same small parameter. In $v_{1}-v_{j}$ geometry the quasi-linear diffusion coefficients are dependent on $v_{1}$ only to first order in $k_{3} / k_{1}$. Physically this occurs because the instability $\left(\omega-L \Omega_{\alpha}=k_{1} v_{A}\right.$ and $\left.k_{1}<<k_{1}\right)$ acts on the perpendicular energy.

The instability is favored at $r+0$ where the fusion rate is maximum. Then 
unstable harmonic numbers permitted in a tokamak magnetic field collapse to a single integer ${ }^{6}$; therefore, the diffusion coefficients are restricted to the sinile harmonics, and to zero order in $k_{1} / k_{1}$ :

with

$$
D_{\perp} \simeq k_{11} v_{\perp} L \Omega_{\alpha}\{\ldots\} \mid ; \quad D_{x} \simeq \frac{1}{2} k_{11}^{2} v_{\perp}^{2} \quad\{\ldots\} ; D_{11} \simeq \frac{1}{3} \frac{k_{11}^{3} v_{\perp}^{3}}{L \Omega_{\alpha}} \quad\{\ldots\} \text {; }
$$

$$
\{\ldots\}=\frac{v_{A}^{2}}{v_{\perp}^{2}} E_{r}^{2} J_{L}^{2}+E_{\theta}^{2}\left(J_{L}^{\prime}\right)^{2} !
$$

The ordering of the diffusion coefficients is found to be:

$$
D_{\perp}: D_{x}: D_{11} \simeq 1: \frac{1}{2} 0\left(\frac{k_{11}}{k_{\perp}}\right): \frac{1}{3} \circ\left(\frac{k_{11}^{2}}{k_{\perp}^{2}}\right)
$$

FMS Wave Growth and Damping--Next we require an expression for the aipha growth rate, $Y_{\alpha}$, and $p$ lasma damping of the wave. Our derivation generalizes prior results ${ }^{3}$ for propagation in the $k_{\perp}$ plane $a t$ an arbitrary angle to the radial vector, and also includes the effect of relative polarization between the radial and poloidal electric fields vs. w. Neglecting terms proportional to $k_{n}$ we obtain:

$$
\gamma_{\alpha} \simeq \frac{-1}{\Delta^{\prime}\left(1+p_{r \theta}^{2}\right)} \frac{\omega_{p \alpha}^{2}}{k_{\perp}^{2}} \int d \vec{v} \frac{1}{v_{\perp}} \frac{\partial \hat{f}_{\alpha}}{\partial v_{\perp}} R\left(v_{\perp}, \omega\right)
$$

and

with

$$
R\left(v_{\perp}, \omega\right)=\sum \frac{L \Omega_{\alpha 0}}{\omega} \frac{\left[J_{L}^{2}+o_{r \theta}^{2} \frac{v_{\perp}^{2}}{v_{A}^{2}}\left(J_{L}^{\prime}\right)^{2}\right]}{\sqrt{\varepsilon^{2}-\left[1-\frac{L \Omega_{\alpha 0}}{\omega}\right]^{2}}}
$$

$$
\frac{\omega}{\Omega_{\alpha}}(1-E)<L<\frac{\omega}{\Omega_{\alpha}}(i+E)
$$

Dependence on $v_{g}$ ir: Eqs. (5) appears only in the normalized alpha distribution function $\mathrm{f} \equiv \mathrm{f}_{\alpha} / \mathrm{n}_{\alpha}$. If the instability operates predominantly along $v_{1}$, we may integrate out the $v_{y}$ dependence. Such a condition exists for the isotropic alpha source without loss cones, which to a first approximation is assumed here.

Threshold Studies--We define threshold fraction, $\eta_{\tau}$, di the ratio of alpha-toelectron density at marginal stability, i.e., when the sum of the alpha-driven growth and damping rates equal zero. He assume the device parameters (major and minor radi $i$ and magnetic field), radial position and wave frequency are fixed and $n_{\tau}$ is computed vs. density and ion iemperature, giving threshold contours in the $n_{e^{-}} T_{j}$ plane. Typical results for a demonstration-sized reactor $\left(\mathrm{FED}^{7}\right)$ are presented in Fig. 1. The threshold contours are incremented by units of -1 since we have plotted $\log _{10}\left(n_{\tau}\right)$. The heavy outer boundary labeled " 0.0 " is defined as the limit outside of which the Fis wave is stable. At a fixed $n_{e}, n_{\tau}$ increases with an increased $T_{j}$ due to Doppler broadening of the alpha source function. Strong variations in $n_{T}$ along the $n_{e}$ axis at tixed $T_{j}$ arise from successive tuning and detuning due to the dependence of the yrowtin and damping rates $\left(r_{\alpha}\right)$ on the Alfuén speed. We find that the instability 
heating is strongest where the threshcld fraction is a minimum $(\sim 10 \mathrm{keV}$ in Fig. 5). Thus, the effect will be strongest for a low $\langle\beta\rangle$ device, where the magnetic field remains relatively strong on axis.

One-Dimensional Quasi-Linear Approximation--Another objective is to assess reactor level effects. We reduce the 2-D quasi-linear diffusion to 1-D with mild restrictions. The final set of 1-D quasi-linear equations is solved using a code package described elsewhere. 6 We use representative reactor parameters: 7 a toroidal magnetic field of $42 \mathrm{kG}$ on axis, $\mathrm{ne} \sim 4.75 \times 10^{13} \mathrm{~cm}^{-3}$ at $5 \mathrm{~cm}$ and $T_{e} \sim T_{j} \sim 10 \mathrm{keV}$. Then $n_{\tau} \sim 3.55 \times 10^{-6}$ for $\omega=6 \Omega_{\alpha}$ and the total wave damping rate is $Y_{d}=437 \mathrm{sec}^{-1}$. The enhancement factor, $P_{i v}$, is the ratio of the time averaged alpha ion heating rate in the presence of the instability vs. that with classical slowing. For present plasma conditions the maximum piv is $\sim 1.3$.

A typical time history is shown in Fig. 2. The total growth rate Y is plotted along with the wave energy density and the response of $P_{i v}$. At $t_{i m e}$ $\tau=0, \gamma_{T}=0$ and the wave electric field $E_{1}$ is set arbitrarily low so that quasilinear diffusion is negligible. As the alpha densities increases, $Y_{T}$ increases since the damping rate (dependent on the background plasma conditions? remains constant. Then, $E_{1}$ grows exponentially until $D_{1}$, which depends on $E_{1}$, becomes large enough to change the alpha profile. As the profile flattens, the growth rate which depends on the slope in $f_{\alpha}\left(v_{j}\right)$ drops. As the instability continues into saturation, $f_{\alpha}\left(v_{1}\right)$ diffuses sufficiently that $\gamma_{\alpha}<-\gamma_{d}$ and the wave decays via cyclotron and collisional damping. After the distribution collapses, the process begins anew and repeats itself after $\sim 15 \mathrm{~ms}$.

Reactor Simulation--We use dąta from the lowest "lobes" of the contours in Fig. l (j.e., at ne $\left.25 \times 10^{3} \mathrm{~cm}^{-3}\right)$ to examine effects in a demonstration-type reactor. The data were incorporated in $11 / 2-0$ tokamak transport code and a typical result is shown in Fig. 3 . Contours of the auxiliary power ( $P$ aux)
required to hold a given $\langle T\rangle$ are plotted. Tre instability occurs in the shaded region, $i . e$. , during the startup phase at moderate density-temperature. The rather restricted instability region is encouraying from a control point of view and could be avoided with an appropriate startup trajectory.

Summary and Conclusion--Generally the threshold is smallest for relatively low electron density, ion cemperature, and $\beta$, although values do extend over a fairly wide range of parameters ( $F$ ig. 1). Furthermore, cf Fig. 2, when the instability is triggered, a significant collapse of the alpha distribution occurs. A repeated buildup and collapse is observed with a period of $\sim 15 \mathrm{~ms}$. Since the energy fluctuation is $<1 \%$ of the background plasma energy, this is not viewed as disruptive. In fact, the increase in alpha energy transferred to background ions (versus electrons) is desirable. Also, the reduction in the time-averaged alpha pressure would be beneficial. However, cf Fiy. 3 , the reyion where the instability occurs is quite isolated.

Acknowledgments--Work sponsored by the U.S. DUE contract DEACU2 $76 E T b 2040$. Contributions by S. K. Ho, Univ. of Illinois, art recognized.

References--[1] A. B. Mikhailovskii, Proc. of the 1979 Intern. School of Plasma Physics, Varenna, Italy, Aug.27-Sept. 8, 1979. [2] A. B. Mikhailovskii, et al. S Soviet J. of Plasma Phys., 5:2, 173 (1979). [3] D. J. Sigmar, Workshop on Physics of Plasmas Near Thermonuclear Conditions; Varenna, Italy, Aug. $27-$ Sept. 7, 1979. [4] W. R. Sutton, D. J. Sigmar, and G. H. Miley, Fusion Technology, 7, 374 (1985). [5] A. I. Akhiezer, et al., Plasma Electro-aynamics, Pergamon Press, New York, 1975, Vol. 2, 67. [6] V. M. Baraakov, A. B. 
Mikhailovskii, Soviet J. of Plasma Phys., 3:2, 121 (1977). [7] C. A. Flanagan, et al., Report UKML/TM-7777, Uak Ridye National Laboratory, Dak Ridge, TN, May 1981. [8] W. R. Sutton, Report C00-2218-251, Fusion Studies Laboratory, Univ. of Illinois, Urbana, IL, June 1982.

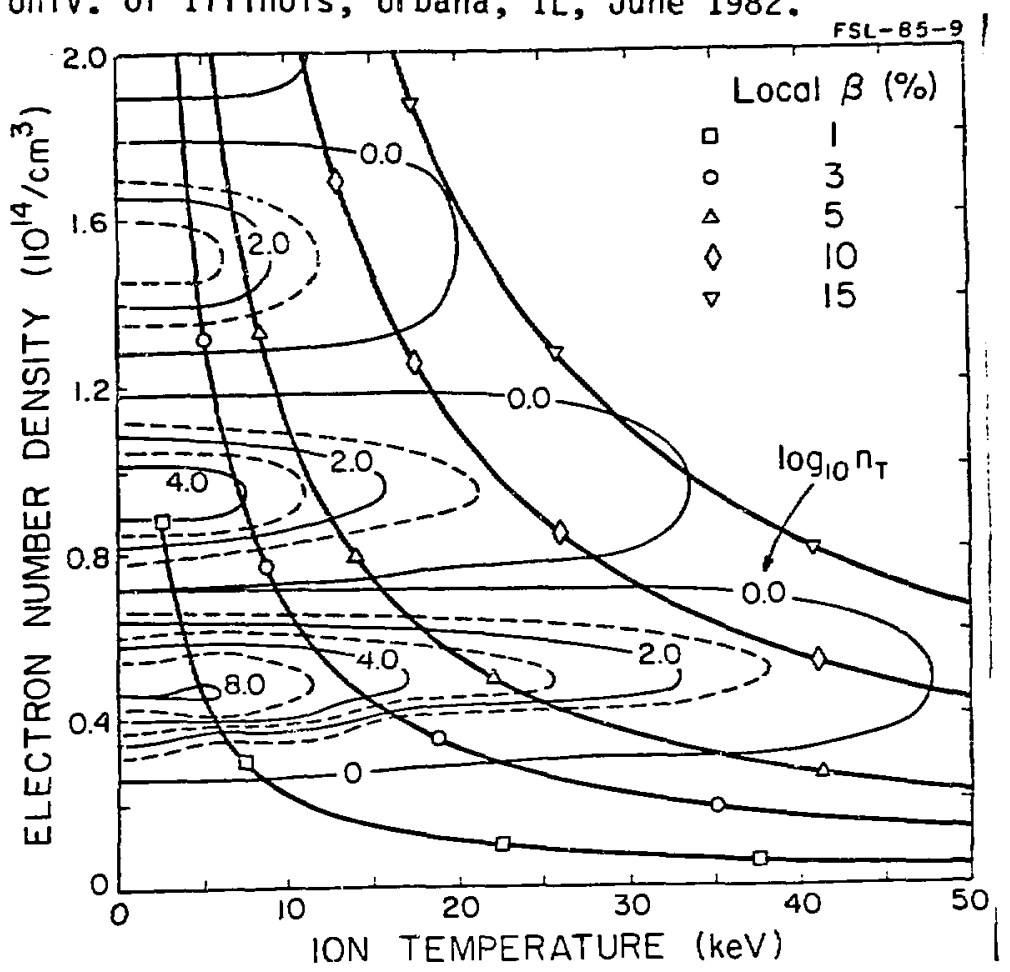

Fig. 1. Alpha threshold fraction $\left(n_{T}\right)$ contours for the FMS wave in the FED: $\omega=6 \Omega_{\alpha}=k_{\perp} v_{A}, k_{\|} \ll k_{1}, B_{0}=42 \mathrm{kG}, r=10 \mathrm{~cm}$.

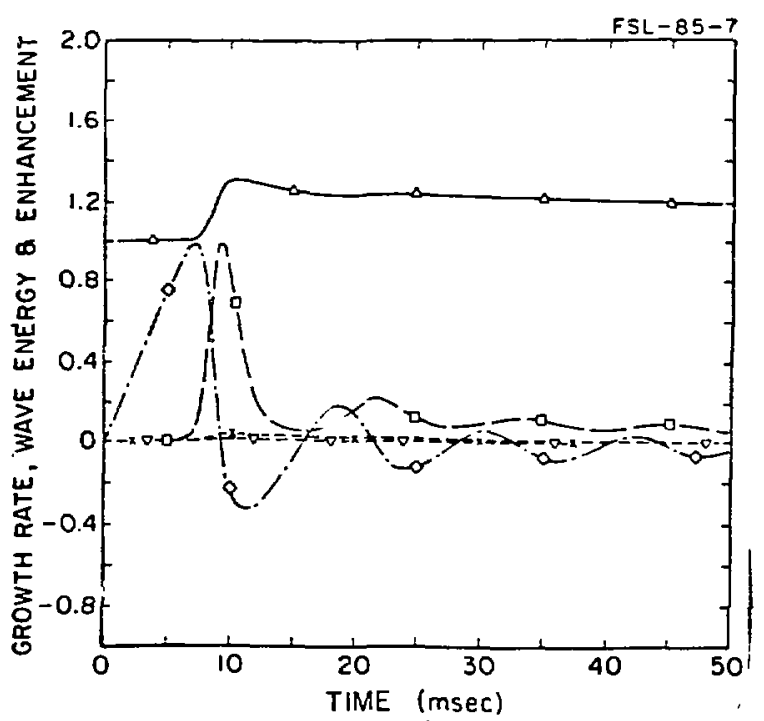

Fig. 2. Time histories of instabiity, [0]=total growth rate $\gamma_{T}=\gamma_{\alpha}{ }^{+} \gamma_{D}$, [a] =wave energy $W_{E(\tau)}, \nabla=$ density, $X=$ relative error in system energy, $\Delta=e n-$ hancement factor $p_{i v}$. The normalizations are $\log _{10}\left(\max . W_{E}\right)=1.992$ and $\log _{10}\left(\max \cdot \gamma_{T}\right)=3.022$

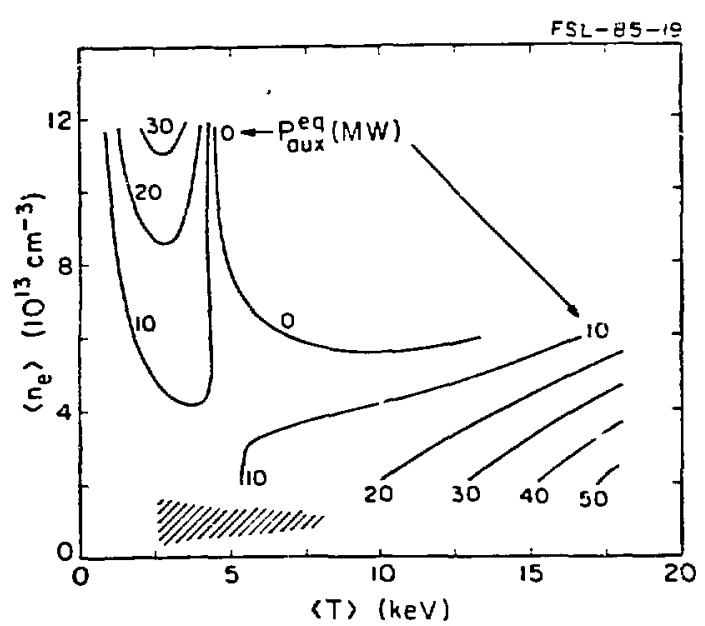

Fig. 3. External power $p_{\text {aux required }}^{\text {eq }}$ to hold a given $\langle n\rangle-\langle T\rangle$ operating point using Alcator scaling and $\mathrm{B}_{0}=$ $42 \mathrm{kl}$. Alpha instability occurs in the shaded reyion. 